\title{
A Turn-On Fluorescent Sensor for Glutathione Based on Bovine Serum Albumin-Stabilized Gold Nanoclusters
}

\author{
Yan Qiu, ${ }^{1,2}$ Jianlin Huang $\mathbb{D}^{1}{ }^{1}$ and Li Jia ${ }^{1}$ \\ ${ }^{1}$ Ministry-of-Education Key Laboratory for the Synthesis and Application of Organic Functional Molecules \& \\ College of Chemistry and Chemical Engineering, Hubei University, Wuhan 430062, China \\ ${ }^{2}$ Yunnan Branch of China National Geological Center of Building Materials Industry, Kunming 650118, China \\ Correspondence should be addressed to Jianlin Huang; jlhuang@hubu.edu.cn
}

Received 30 September 2018; Accepted 4 November 2018; Published 2 December 2018

Academic Editor: Neil D. Danielson

Copyright (C) 2018 Yan Qiu et al. This is an open access article distributed under the Creative Commons Attribution License, which permits unrestricted use, distribution, and reproduction in any medium, provided the original work is properly cited.

\begin{abstract}
A fluorescence sensor for the detection of glutathione based on the fluorescence recovering of the bovine serum albumin-stabilized gold nanoclusters is reported. This study indicates that glutathione restores the copper-ion-quenched fluorescence by coordinating the bound copper ion in the bovine serum albumin molecule used for stabilizing the gold nanocluster through its sulfydryl. Under the experimental conditions, the fluorescence response showed a linear relationship with the concentration of glutathione over the range from $10 \mu \mathrm{M}$ to $400 \mu \mathrm{M}$. The fluorescence sensor successfully detected glutathione in commercial drug products.
\end{abstract}

\section{Introduction}

Development of glutathione (GSH) assay methods has received attention due to its diverse functions in organisms and extensive market prospects. GSH, an important nonenzymatic antioxidant, is found in almost all cell types. GSH protects cells from damage of reactive oxygen species such as hydroxyl radical, hydrogen peroxide, and lipid peroxides, directly by eliminating free radicals, and indirectly by serving as a cofactor for glutathione peroxidase $[1,2]$. GSH also participates in other physiological processes such as control of cell proliferation and nucleotide metabolism [3, 4]. Based on its essential role in the health of organisms, GSH is used in clinic to treat kinds of diseases such as liver disease and uremia and reduce the side effects correlated with chemoradiotherapy.

Many analytical methods, such as high performance liquid chromatography, capillary electrophoresis, fluorophotometry, and electrochemistry, have been developed for detection of GSH [5-8]. Among these methods, fluorophotometry has advantages over the other techniques at sensitivity, simplicity, and costs. In recent years, fluorescent probes for detection of GSH have been designed and investigated for overcoming the disadvantages of traditional fluorometric assays [9-12]. Although these fluorescent probes successfully detected GSH from various samples, including aqueous solutions, human serum, bovine serum album (BSA), and liposome, they suffered from complicated and tedious synthesis procedures.

Bovine serum albumin-protected fluorescent gold nanoclusters (AuNCs-BSA) reported by Xie et al. have given rise to research interest in sensing applications owing to the advantages of facile preparation, high fluorescence quantum yield ( $\sim \%)$, favorable photostability, and good biocompatibility [13]. Xie's research group developed a simple label-free method for the selective and sensitive detection of $\mathrm{Hg}^{2+}$ based on fluorescence quenching of AuNCs-BSA triggered by $\mathrm{Hg}^{2+}$ $\mathrm{Au}^{+}$interactions [14]. Liu et al. reported a AuNCs-BSA-based fluorescent sensor for the recognition and determination of cyanide in aqueous solution, which was based on the fluorescence quenching of AuNCs-BSA induced by the Elsner reaction between cyanide and gold atoms of AuNCs-BSA [15]. Durgads et al. demonstrated the AuNCs-BSA can be used as a selective fluorescence "turn-off" sensor for $\mathrm{Cu}^{2+}$ in live cells based on fluorescence quenching of AuNCs-BSA resulting from intersystem crossing of the excited electron from the gold cluster stimulated by the bound $\mathrm{Cu}^{2+}$ in the BSA molecule [16]. Their paper also showed that the 
copper-ion-quenched emission was reversible with a copper chelator glycine.

A previous study demonstrated that the fluorescence of GSH-capped gold nanoparticles was quenched by $\mathrm{Cu}^{2+}$ due to the complexation between $\mathrm{Cu}^{2+}$ and GSH [17]. Thus, we assumed that GSH might be able to retrieve the copperion-quenched fluorescence of AuNCs-BSA by coordinating $\mathrm{Cu}^{2+}$. GSH was found to be much more effective than glycine on restoring the fluorescence quenched by copper ions in our study. Thus, we have developed a fluorescence "turn-on" sensor for GSH based on the AuNCs-BSA-Cu system.

\section{Materials and Methods}

2.1. Reagents. Glutathione (98\%), HEPES (99\%), and amino acids $(\geq 98 \%)$ were purchased from Aladdin Biochemical Technology Co., Ltd. (Shanghai, China). $\mathrm{HAuCl}_{4}$ (AR), bovine serum albumin (BR), $\mathrm{NaOH}$ (AR), and metal nitrates (AR) were purchased from Sinopharm Chemical Reagent Co., Ltd. (Shanghai, China). Reduced glutathione for injection and reduced glutathione tablets were from YaoPharma Co., Ltd. (Chongqing, China). Ultrapure water with $18.2 \mathrm{M} \Omega \cdot \mathrm{cm}$ resistivity was used for preparing the solutions.

2.2. Preparation of AuNCs-BSA. The AuNCs-BSA was synthesized based on a modified Xie method [13]. In brief, $\mathrm{HAuCl}_{4}$ solution $(25 \mathrm{~mL}, 10 \mathrm{mM})$ was mixed with BSA solution $\left(25 \mathrm{~mL}, 30 \mathrm{mg} \cdot \mathrm{mL}^{-1}\right)$ under vigorous stirring at $37^{\circ} \mathrm{C}$. Two minutes later, $\mathrm{NaOH}$ solution $(3 \mathrm{~mL}, 1 \mathrm{M})$ was introduced, and the mixture was incubated at $37^{\circ} \mathrm{C}$ for $12 \mathrm{~h}$.

2.3. Detection of GSH. For fluorescent detection of GSH, varying volumes of $10 \mathrm{mM}$ GSH solutions were mixed with the AuNCs-BSA solution containing $\mathrm{Cu}^{2+}$ which was prepared by adding $30 \mu \mathrm{L} 10 \mathrm{mM} \mathrm{Cu}^{2+}$ solution to $250 \mu \mathrm{L}$ AuNCs-BSA solution, and the mixtures were diluted to $5 \mathrm{~mL}$ with HEPES buffer $(\mathrm{pH}=7.2)$. Fluorescence emission spectra of the as-prepared solutions were measured under $480 \mathrm{~nm}$ excitation.

2.4. Sample Preparation. A bottle of reduced glutathione powder for injection was dissolved and diluted to $100 \mathrm{~mL}$ with ultrapure water. After four reduced glutathione tablets were ground, the powder was dissolved in ultrapure water and filtered. The filtrate was finally diluted to $100 \mathrm{~mL}$ with ultrapure water.

\section{Results and Discussion}

3.1. Mechanism for Fluorescence Recovering of AuNCs-BSA. The deep brown solution of AuNCs-BSA emits a red fluorescence under $480 \mathrm{~nm}$ excitation. The fluorescent emission peak at $648 \mathrm{~nm}$ was found to disappear upon addition of $300 \mu \mathrm{M} \mathrm{Cu}^{2+}$. It was further observed that the AuNCs-BSA solution containing $\mathrm{Cu}^{2+}$ emitted strong fluorescence again after treatment with of 1.6 mM GSH (Figure 1).

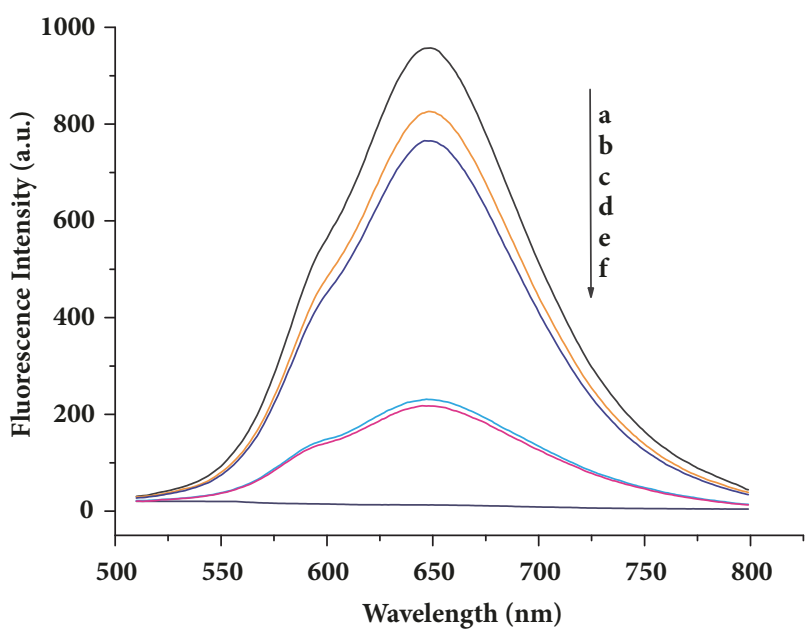

FIgURE 1: Fluorescence emission spectra of AuNCs-BSA in the absence (a) and presence of $\mathrm{Cu}^{2+}(\mathrm{f})$. Fluorescence recovery from the quenched AuNCs-BSA by adding of GSH (b), cysteine (c), glycine (d), and glutamic acid (e).

The fluorescence quenching of AuNCs-BSA in the presence of $\mathrm{Cu}^{2+}$ was attributed to the binding of $\mathrm{Cu}^{2+}$ on to the BSA used for stabilizing the gold nanocluster, which enabled the paramagnetic $\mathrm{Cu}^{2+}$ to prompt intersystem crossing of the excited electron from the gold cluster and consequently decreased the fluorescence intensity [16]. A control experiment showed that GSH had no influence on the fluorescence spectrum of AuNCs-BSA in the absence of $\mathrm{Cu}^{2+}$, indicating that the fluorescence recovery induced by adding GSH to the AuNCs-BSA-Cu system resulted from the interaction between $\mathrm{GSH}$ and $\mathrm{Cu}^{2+}$. GSH, a natural tripeptide that consists of glutamate, cysteine, and glycine, contains various coordinating function groups such as carboxyl, amido, sulfydryl, and acylamino, which facilitates its molecules to form complexes with metal ions. GSH was replaced by glutamic acid, cysteine, and glycine, respectively, to observe the change in fluorescence properties of the AuNCs-BSA-Cu system and identify the binding site on GSH for $\mathrm{Cu}^{2+}$. It is apparent in Figure 1 that the fluorescence intensity restored by cysteine was close to that by GSH at the same concentration and much stronger than that by glycine or glutamic acid. Considering the facts that $\mathrm{Cu}^{2+}$ is characterized by a strong affinity for $\mathrm{SH}$ residues and among the three amino acids constituting GSH only cysteine has a sulfydryl, we speculate that GSH recovers the copper-quenched fluorescence of AuNCs-BSA by coordinating the bound $\mathrm{Cu}^{2+}$ in the BSA molecule used for stabilizing the gold nanocluster through its sulfydryl.

3.2. Optimization of Conditions for GSH Sensing. Concentration dependent effects of AuNCs-BSA and $\mathrm{Cu}^{2+}$ on the detection of GSH were investigated. High concentrations of $\mathrm{Cu}^{2+}$ were required for high fluorescence quenching efficiency at high concentrations of AuNCs-BSA, which means low detection sensitivity for GSH. On the other hand, too low a concentration of $\mathrm{Cu}^{2+}$ would increase background 


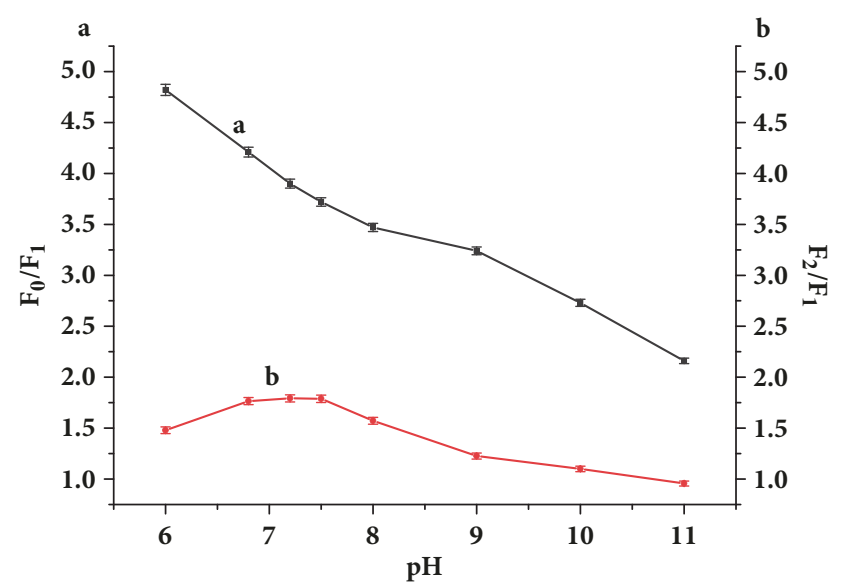

FIGURE 2: Fluorescence quenching efficiency of $\mathrm{Cu}^{2+}$ (a) and fluorescence recovering efficiency of GSH (b) at different $\mathrm{pH}$ values.

fluorescence and narrow the allowing quantitative range of GSH due to low fluorescence quenching ability. In a solution with a total volume of $5 \mathrm{~mL}, 250 \mu \mathrm{L}$ AuNCs-BSA and $60 \mu \mathrm{M}$ $\mathrm{Cu}^{2+}$ were finally selected for GSH sensing.

The acid effect on the sensing system was studied over a $\mathrm{pH}$ range from 6 to 11 . When the $\mathrm{pH}$ value increased in the tested range, diminutive change in the fluorescence intensity of AuNCs-BSA was observed, whereas the fluorescence intensity of the AuNCs-BSA in the presence of $\mathrm{Cu}^{2+}$ increased, indicating the fluorescence quenching efficiency of $\mathrm{Cu}^{2+}$ decreased with increasing of the $\mathrm{pH}$ value. It was also observed that the fluorescence recovering efficiency of GSH changed with the $\mathrm{pH}$ value. The fluorescence quenching and recovering efficiency are represented with $\mathrm{F}_{0} / \mathrm{F}_{1}$ and $\mathrm{F}_{2} / \mathrm{F}_{1}$ respectively, where $\mathrm{F}_{0}$ and $\mathrm{F}_{1}$ correspond to the fluorescence intensity of the AuNCs-BSA in the absence and presence of $\mathrm{Cu}^{2+}$, respectively. $\mathrm{F}_{2}$ represents the fluorescence intensity of the AuNCs-BSA in the presence of $\mathrm{Cu}^{2+}$ and GSH. As shown in Figure 2, the fluorescence recovering efficiency of GSH is stabilized and maximized at physiological $\mathrm{pH}$. The HEPES buffer solution was finally employed to adjust the $\mathrm{pH}$ of solutions used in the measurement to 7.2.

Time-dependent fluorescence signals of the sensing system were observed. The change in fluorescence properties of AuNCs-BSA in the absence and presence of $\mathrm{Cu}^{2+}$ was not obvious within 30 minutes. However, the fluorescence intensity of the AuNCs-BSA in the presence of $\mathrm{Cu}^{2+}$ and GSH slowly decreased with time, and thus the fluorescence recovering efficiency decreased with time (Figure 3). Therefore, the fluorescence of the sensing system should be measured immediately upon adding GSH to the solution of AuNCs$\mathrm{BSA}$ in the presence of $\mathrm{Cu}^{2+}$.

3.3. Selectivity and Sensitivity for GSH Sensing. Although the presence of $\mathrm{Pb}^{2+}, \mathrm{Co}^{2+}$, or $\mathrm{Ni}^{2+}$ with the same concentration of $\mathrm{Cu}^{2+}(60 \mu \mathrm{M})$ showed a quenching effect on the fluorescence of the AuNCs-BSA, their quenching efficiencies were much lower than that of $\mathrm{Cu}^{2+}$ (Figure 4). The degree of

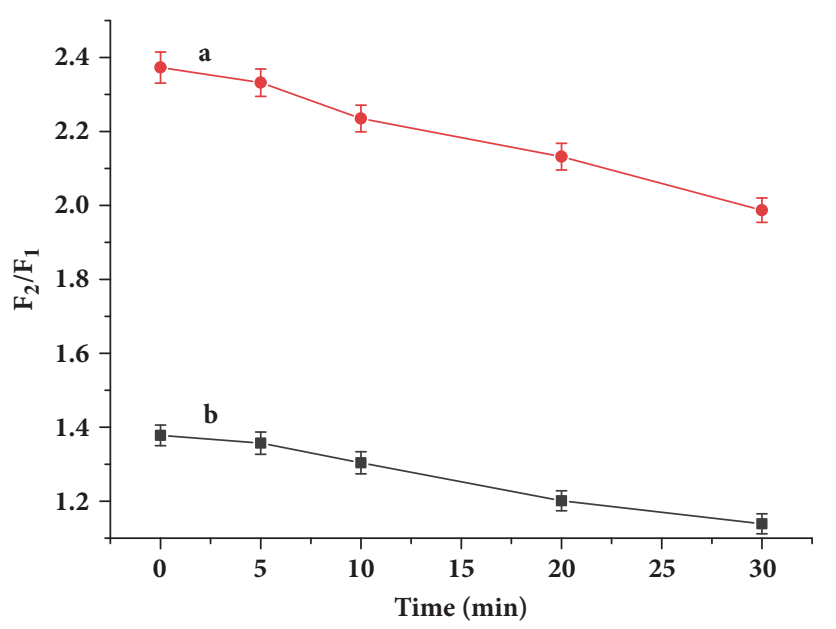

FIgURE 3: Time-dependent fluorescence recovering efficiency of $200 \mu \mathrm{M}$ GSH (a) and $40 \mu \mathrm{M}$ GSH (b).

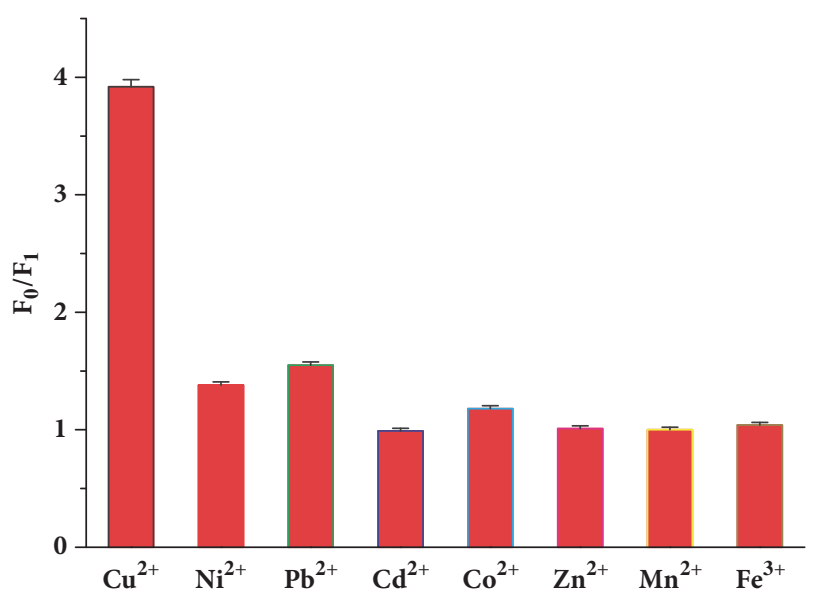

FIGURE 4: Fluorescence quenching effect of metal ions $\left(\mathrm{F}_{0}\right.$ and $\mathrm{F}_{1}$ are the fluorescence intensity of the AuNCs-BSA in the absence and presence of metal ions, respectively.).

interference of other metal ions, including $\mathrm{K}^{+}, \mathrm{Ca}^{2+}, \mathrm{Mg}^{2+}$, $\mathrm{Zn}^{2+}, \mathrm{Cd}^{2+}, \mathrm{Mn}^{2+}$, and $\mathrm{Fe}^{3+}$, for the detection of GSH was further investigated. On the basis of a relative error range from $-5 \%$ to $5 \%$ in detecting $50 \mu \mathrm{M}$ GSH, the tolerance concentrations were as follows: $1 \mathrm{mM}$ for $\mathrm{K}^{+}, \mathrm{Ca}^{2+}, \mathrm{Mg}^{2+}$, $500 \mu \mathrm{M}$ for $\mathrm{Zn}^{2+}, \mathrm{Mn}^{2+}, \mathrm{Cd}^{2+}$, and $100 \mu \mathrm{M}$ for $\mathrm{Fe}^{3+}$. Some amino acids were also used to evaluate the selectivity of the sensing system. As shown in Figure 5, only cysteine could result in significant fluorescence recovery of the AuNCs-BSA, whereas no obvious changes in the quenched fluorescence were observed in the presence of other amino acids such as glycine, lysine, proline, glutamic acid, tryptophan, and phenylalanine at the same concentration of GSH $(50 \mu \mathrm{M})$.

Under the optimum detection conditions, the relationship between the fluorescence recovering efficiency $\left(\mathrm{F}_{2} / \mathrm{F}_{1}\right)$ and the concentration of GSH over the range from $10 \mu \mathrm{M}$ to $400 \mu \mathrm{M}$ could be expressed by a linear equation $\left(\mathrm{R}^{2}=0.996\right)$, 
TABLE 1: Determination of GSH in practical samples $(n=5)$.

\begin{tabular}{lccccc}
\hline Samples & Detected $/ \mu \mathrm{M}$ & Added $/ \mu \mathrm{M}$ & Total found $/ \mu \mathrm{M}$ & Recovery $/ \%$ & RSD $/ \%$ \\
\hline GSH tablets & 155.1 & 80.0 & 231.6 & 98.5 & 3.9 \\
GSH powder & 96.6 & 50.0 & 148.5 & 101.3 & 2.6 \\
\hline
\end{tabular}

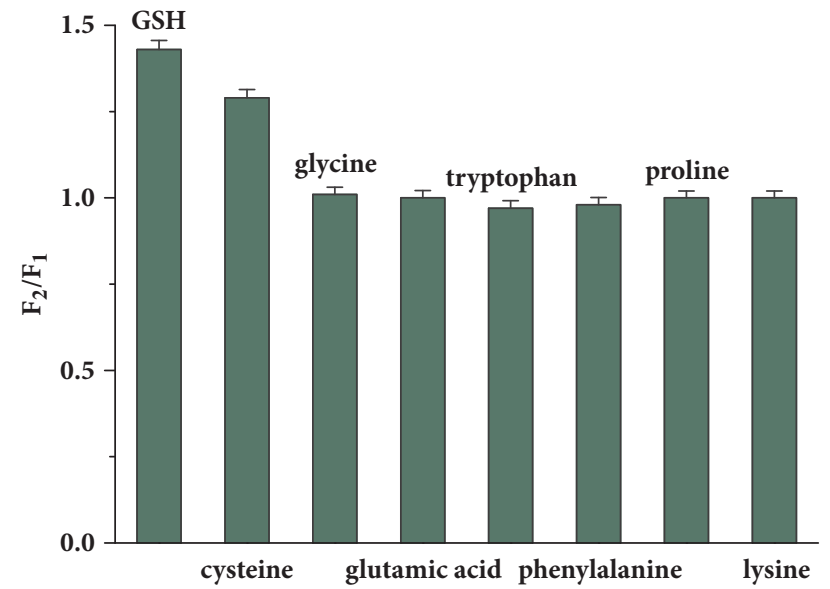

FIGURE 5: Selectivity of the sensor for GSH over amino acids $\left(\mathrm{F}_{2}\right.$ is the fluorescence intensity of the AuNCs-BSA in the presence of $\mathrm{Cu}^{2+}$ and GSH or amino acids.).

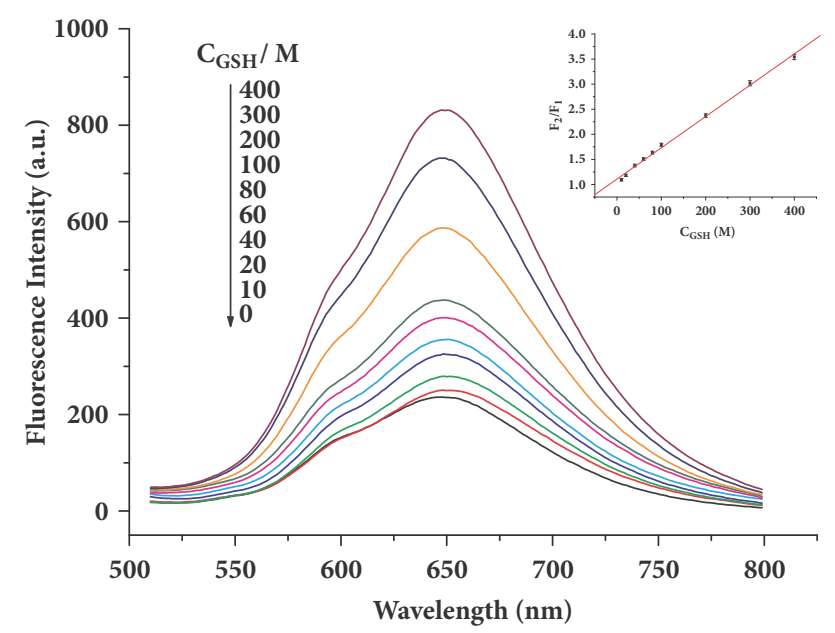

FIGURE 6: Fluorescence recovery from the quenched AuNCs-BSA by adding of various concentrations of GSH. Inset: relationship between the fluorescence recovering efficiency and the concentration of GSH.

$\mathrm{F}_{2} / \mathrm{F}_{1}=0.0063 \mathrm{C}_{\mathrm{GSH}}+1.09$ (Figure 6). The limit of detection for $\mathrm{GSH}$ was calculated to be $1.2 \mu \mathrm{M}$.

3.4. Application. Commercial reduced glutathione tablets and reduced glutathione powder for injection were employed as practical samples to evaluate the applicability of the GSH sensor developed here. The recovery and relative standard deviation obtained with a standard addition method through five parallel tests are presented in Table 1.

\section{Conclusions}

We found that GSH restored effectively the copper-quenched fluorescence from the AuNCs-BSA and therefore develop a new fluorescence "turn-on" sensor for GSH detection. The sensor shows advantages such as fast and sensitive response to GSH, simplicity in preparation and usage, and environmental friendliness. The recovery and precision obtained from commercial GSH drug products indicate the potential application of the GSH sensor.

\section{Data Availability}

The data used to support the findings of this study are available from the corresponding author upon request.

\section{Conflicts of Interest}

The authors declare that there are no conflicts of interest regarding the publication of this paper.

\section{Acknowledgments}

This work was supported by Ministry-of-Education Key Laboratory for the Synthesis and Application of Organic Functional Molecules, Hubei University (no. 2015-KL-009).

\section{References}

[1] A. Prima, K. Y. Hara, A. C. Djohan et al., "Glutathione production from mannan-based bioresource by mannanase/ mannosidase expressing Saccharomyces cerevisiae," Bioresource Technology, vol. 245, pp. 1400-1406, 2017.

[2] J. R. Arthur, "The glutathione peroxidases," Cellular and Molecular Life Sciences, vol. 57, no. 13-14, pp. 1825-1835, 2000.

[3] J. L. García-Giménez, C. Romá-Mateo, G. Pérez-Machado, L. Peiró-Chova, and F. V. Pallardó, "Role of glutathione in the regulation of epigenetic mechanisms in disease," Free Radical Biology \& Medicine, vol. 112, pp. 36-48, 2017.

[4] Y. Chen, H. Dong, D. C. Thompson, H. G. Shertzer, D. W. Nebert, and V. Vasiliou, "Glutathione defense mechanism in liver injury: insights from animal models," Food and Chemical Toxicology, vol. 60, pp. 38-44, 2013.

[5] L. Janeš, K. Lisjak, and A. Vanzo, "Determination of glutathione content in grape juice and wine by high-performance liquid chromatography with fluorescence detection," Analytica Chimica Acta, vol. 674, no. 2, pp. 239-242, 2010.

[6] E. Tsardaka, C. K. Zacharis, P. D. Tzanavaras, and A. Zotou, "Determination of glutathione in baker's yeast by capillary electrophoresis using methyl propiolate as derivatizing reagent," Journal of Chromatography A, vol. 1300, pp. 204-208, 2013.

[7] N. Zhang, W. Ma, D. Han, L. Wang, T. Wu, and L. Niu, "The fluorescence detection of glutathione by $\bullet \mathrm{OH}$ radicals' 
elimination with catalyst of MoS2/rGO under full spectrum visible light irradiation," Talanta, vol. 144, Article ID 15768, pp. 551-558, 2015.

[8] Y. Wang, L. Jiang, Q. Leng, Y. Wu, X. He, and K. Wang, "Electrochemical sensor for glutathione detection based on mercury ion triggered hybridization chain reaction signal amplification," Biosensors and Bioelectronics, vol. 77, pp. 914-920, 2016.

[9] C. Yang, W. Deng, H. Liu, S. Ge, and M. Yan, "Turn-on fluorescence sensor for glutathione in aqueous solutions using carbon dots- $\mathrm{MnO} 2$ nanocomposites," Sensors and Actuators B: Chemical, vol. 216, pp. 286-292, 2015.

[10] L. Wang, X. Chen, and D. Cao, "A novel fluorescence turnon probe based on diketopyrrolopyrrole-nitroolefin conjugate for highly selective detection of glutathione over cysteine and homocysteine," Sensors and Actuators B: Chemical, vol. 244, pp. 531-540, 2017.

[11] Q. Cai, J. Li, J. Ge et al., "A rapid fluorescence "switch-on" assay for glutathione detection by using carbon dots- $\mathrm{MnO} 2$ nanocomposites," Biosensors and Bioelectronics, vol. 72, pp. 3136, 2015.

[12] R. Gui, X. An, H. Su et al., "Rhodamine 6G conjugatedquantum dots used for highly sensitive and selective ratiometric fluorescence sensor of glutathione," Talanta, vol. 94, pp. 295300, 2012.

[13] J. Xie, Y. Zheng, and J. Y. Ying, "Protein-directed synthesis of highly fluorescent gold nanoclusters," Journal of the American Chemical Society, vol. 131, no. 3, pp. 888-889, 2009.

[14] J. Xie, Y. Zheng, and J. Y. Ying, "Highly selective and ultrasensitive detection of $\mathrm{Hg}^{2+}$ based on fluorescence quenching of $\mathrm{Au}$ nanoclusters by $\mathrm{Hg}^{2+}-\mathrm{Au}^{+}$interactions," Chemical Communications, vol. 46, no. 6, pp. 961-963, 2010.

[15] Y. Liu, K. Ai, X. Cheng, L. Huo, and L. Lu, "Gold-nanoclusterbased fluorescent sensors for highly sensitive and selective detection of cyanide in water," Advanced Functional Materials, vol. 20, no. 6, pp. 951-956, 2010.

[16] C. V. Durgadas, C. P. Sharma, and K. Sreenivasan, "Fluorescent gold clusters as nanosensors for copper ions in live cells," Analyst, vol. 136, no. 5, pp. 933-940, 2011.

[17] W. Chen, X. Tu, and X. Guo, "Fluorescent gold nanoparticlesbased fluorescence sensor for $\mathrm{Cu}^{2+}$ ions," Chemical Communications, no. 13, pp. 1736-1738, 2009. 

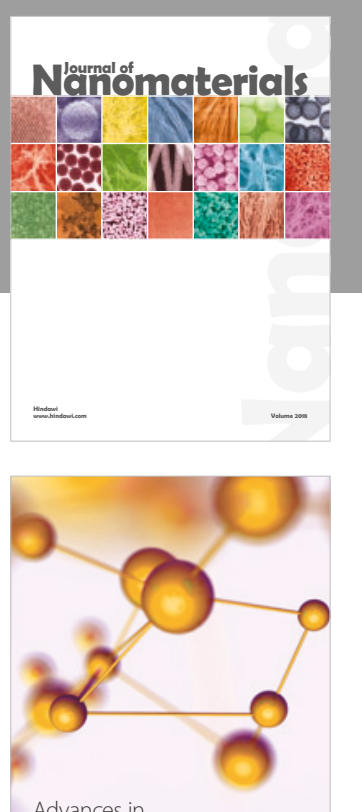

Physical Chemistry
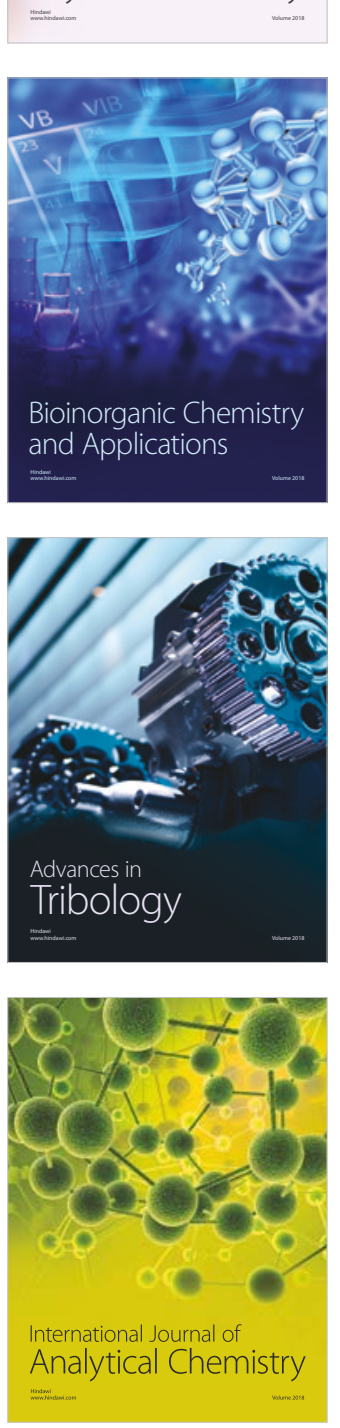

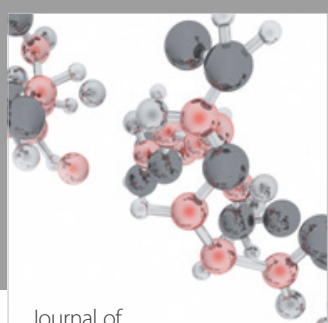

Analytical Methods

in Chemistry

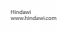

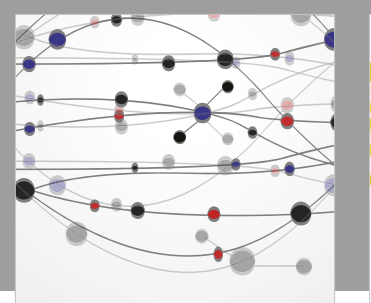

The Scientific World Journal

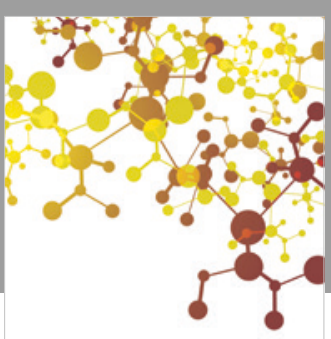

Journal of

Applied Chemistry
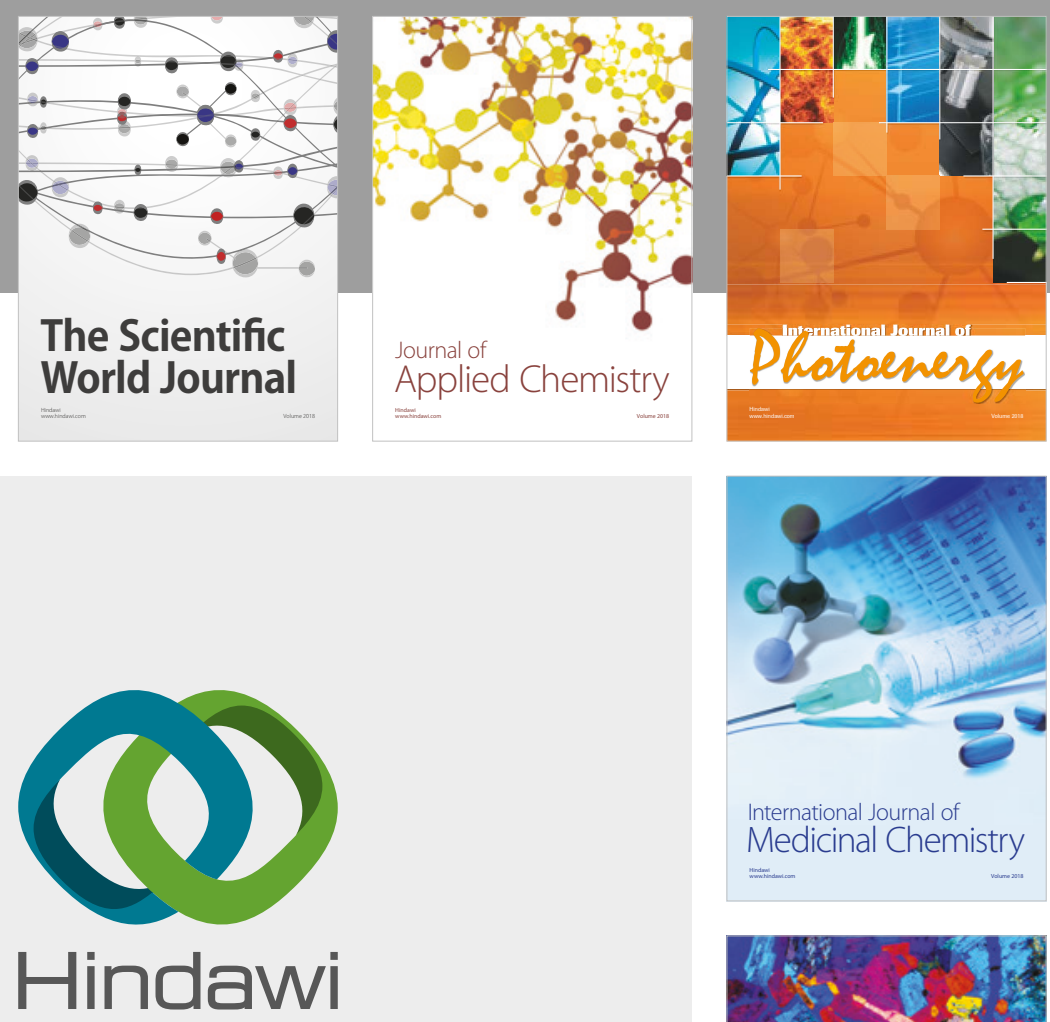

Submit your manuscripts at

www.hindawi.com
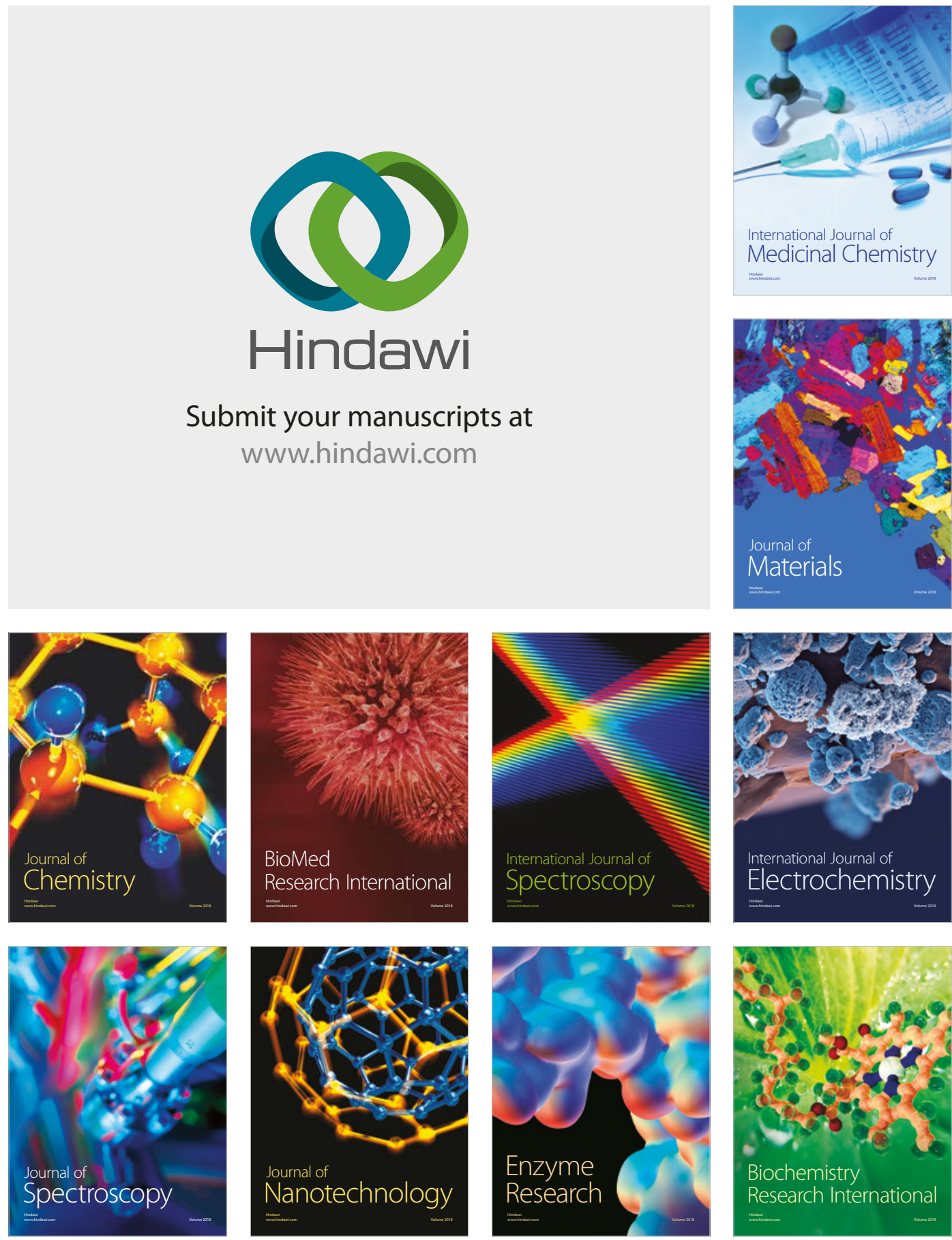
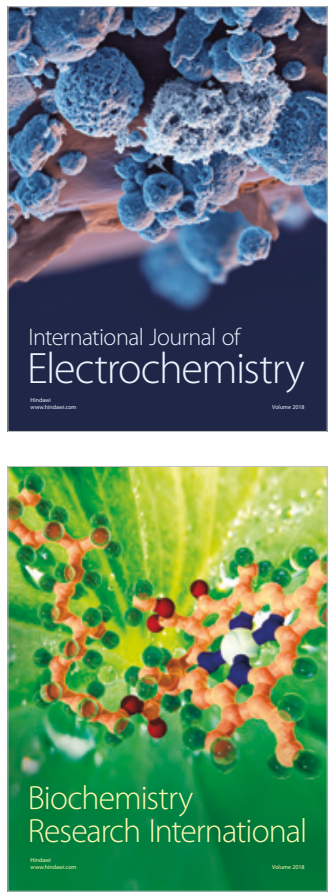